\title{
Efeitos de um Processo de Alfabetização em Informática na Cognição de Idosos
}

\author{
Effects of Digital Literacy Process in Elderly Cognition
}

\author{
Natália Nunes Scoralick-Lempke*, Altemir José Gonçalves Barbosa \\ \& Márcia Maria Peruzzi Elia da Mota \\ Universidade Federal de Juiz de Fora, Juiz de Fora, Brasil
}

\begin{abstract}
Resumo
Para testar a hipótese de que um processo de alfabetização digital melhora as capacidades cognitivas de idosos, mais especificamente as memórias episódica e de trabalho, a meta-memória e a autoeficácia em memória, constituíram-se dois grupos experimentais (GE) com, no total, 22 idosos que participaram de uma Oficina de Inclusão Digital. Esses construtos foram avaliados pré e pós-atividades, sendo que o desempenho do GE foi comparado ao de um grupo controle (GC) com 16 participantes também idosos. Após 19 oficinas de duas horas cada, verificou-se que as alterações nos processos cognitivos dos participantes, observadas na presente investigação, não podem ser atribuídas a esse processo educacional. As limitações do quase experimento e as implicações sociais e científicas dos resultados são analisadas.

Palavras-chave: Envelhecimento, processos cognitivos, alfabetização digital, Gerontologia Educacional.

Abstract

In order to test the hypothesis that digital literacy skills improve the elderly cognitive performance, more specifically episodic and working memory, meta-memory, and auto-efficacy in memory, two experimental groups (EG) were created. The groups were formed with a total of 22 elderly people who participated in a workshop on Digital Inclusion. The constructs were assessed in pre- and post-activities. EG performance was compared to a control group (CG) composed by other 16 elderly participants. After 19 workshops of two hours each, the changes in cognitive processes of the elderly which were observed in this research cannot be assigned to the educational process evaluated. The limitations of the quasi experiment and scientific and social implications of the results are also analyzed.

Keywords: Aging, cognitive processes, digital literacy, Educational Gerontology.
\end{abstract}

O envelhecimento humano envolve modificações substanciais na estrutura física e nas manifestações da cognição, além de alterar a percepção subjetiva dessas transformações. $\mathrm{O}$ declínio de algumas funções intelectuais tem sido considerado inevitável nesse processo (Engelhardt, Laks, Rozenthal, \& Marinho, 1998). No entanto, as perdas delas comumente não afetam as capacidades de forma generalizada e nem ocorre de forma homogênea em todos os indivíduos (Salthouse, 1984). Enquanto algumas tendem a declinar com a idade, outras podem se manter ou até mesmo melhorar em função da experiência de vida de cada indivíduo (Baltes, 1987; Parente \& Wagner, 2006).

De acordo com Shacter (2003), as funções que tendem a se preservar com o envelhecimento são a inteligência cristalizada, a memória implícita, as habilidades motoras

\footnotetext{
* Endereço para correspondência: Universidade Federal de Juiz de Fora, Instituto de Ciências Humanas, Campus Universitário, São Pedro, Juiz de Fora, MG, Brasil 36001-970. E-mail: nataliascoralick@yahoo.com.br, altgonc@uol.com.br e mmotapsi@gmail.com
}

e o aprendizado não-associativo. Dentre as funções que parecem declinar, encontram-se a memória de trabalho e a memória explícita, as habilidades visoespaciais e as funções atentivas e executivas (Damasceno, 1999). Dentre os fatores responsáveis por essas alterações, incluem-se os aspectos genéticos, ambientais e educacionais, a dieta, as atividades diárias, a prática de exercícios físicos e a estimulação cognitiva (Argimon \& Stein, 2005; Pope, Shue, \& Beck, 2003).

A memória é uma função que merece destaque no estudo do envelhecimento por ser uma das funções mais afetadas por esse processo (Mattos et al., 2003). Um percentual expressivo dos idosos queixa-se de problemas dessa ordem (Almeida, 1998). Especificamente, a memória de trabalho e as memórias explícitas parecem ser as mais atingidas no decorrer dos anos (Taussik \& Wagner, 2006). De acordo com Baddeley (2000), a memória de trabalho (ou operacional) é um sistema integrado que permite tanto o processamento ativo, quanto o armazenamento temporal de informação, ambos envolvidos na realização de tarefas cognitivas complexas, tais como o raciocínio, a compreensão e a linguagem. As memórias 
explícitas (ou declarativas), por sua vez, envolvem o pensamento consciente e consiste em um sistema que armazena eventos, fatos ou conhecimentos sobre os quais se pode falar (Lombroso, 2004). Elas incluem as memórias episódica, relativa ao armazenamento de experiências pessoais, e semântica, composta por conhecimentos gerais e consolidada na ausência de referências de tempo e de contexto (Taussik \& Wagner, 2006).

O declínio dos sistemas de memória tem repercussões expressivas para o envelhecimento, interferindo na capacidade de o indivíduo compreender textos, realizar inferências, reconhecer faces e lugares, armazenar informações novas e resgatá-las, o que, por sua vez, gera prejuízos ocupacionais e sociais (Abreu, Forlenza, \& Barros, 2005; Souza \& Chaves, 2005). Devido à função central que a memória desempenha nos processos cognitivos, suas alterações têm implicações no convívio social, no autocuidado e na capacidade de planejamento, podendo acarretar perdas importantes na autonomia e na qualidade de vida do indivíduo (Abreu et al., 2005; Carvalho, Neri, \& Yassuda, 2010).

As alterações de memória também constituem importantes indicadores de doença no envelhecimento, podendo ocasionar demências ou comprometimento cognitivo leve (CCL). De acordo com o DSM-IV (American Psychiatric Association [APA], 1995), a demência é uma doença neurodegenerativa progressiva e sua característica essencial é o desenvolvimento de múltiplos déficits cognitivos, que incluem comprometimento da memória e pelo menos uma das seguintes perturbações cognitivas: afasia, apraxia, agnosia ou perturbação do funcionamento executivo. Os déficits cognitivos devem ser suficientemente severos para comprometer o funcionamento ocupacional ou social e representar um declínio em relação a um nível anteriormente superior de funcionamento. Por sua vez, o CCL é um estado intermediário entre o envelhecimento normal e a demência, caracterizado pelo déficit de memória sem que haja o preenchimento dos critérios para demência (Hamdan, 2008). Segundo Bottino e Moreno (2006), apesar de ele não ser considerado doença, pessoas com CCL apresentam alto risco de desenvolver demência.

As atitudes e crenças dos idosos em relação ao próprio desempenho cognitivo também podem ter repercussões em atividades de memória, influenciando a maneira pela qual a tarefa é realizada, bem como seu desempenho final (Hertzog et al., 1998, citado por Yassuda, Lasca, \& Neri, 2005). Dessa forma, a existência de uma crença em um déficit de memória pode afetar negativamente o desempenho em tarefas dessa natureza. Na literatura, esse conjunto de sentimentos e ideias sobre a memória é denominado metamemória.

A autoeficácia em memória é outro aspecto que vem sendo estudado e é considerado por alguns autores como um componente da metamemória. De acordo com Bandura (1977), autoeficácia se refere à avaliação do indiví- duo sobre sua capacidade de realizar uma determinada tarefa e envolve sempre um objeto específico. Na presente investigação, o objeto da autoeficácia é a memória. A autoeficácia em memória é um construto importante no estudo do envelhecimento, uma vez que, se ela estiver baixa, pode ajudar a explicar os problemas de memória em idosos (Yassuda et al., 2005).

Ainda que certos déficits de memória sejam considerados normais em pessoas com mais de 60 anos, a criação de estratégias de promoção da saúde cognitiva é de fundamental importância, a fim de que patologias possam ser prevenidas na velhice. Alguns estudos têm comprovado os benefícios de uma vida ativa na saúde de idosos. Argimon e Stein (2005), por exemplo, em estudo longitudinal com idosos octogenários, constataram que o número de atividades realizadas pelos indivíduos pesquisados, tais como o envolvimento com amigos, com a igreja e com a cultura local, parecem ser fatores protetores em relação ao declínio de funções mentais. A realização de atividades físicas durante a vida também tem sido considerada um importante fator de proteção das capacidades cognitivas nessa população (Antunes et al., 2006; Argimon \& Stein 2005).

De forma análoga, as atividades intelectuais parecem exercer papel essencial na manutenção da cognição no envelhecimento (Gage, 2003). Dessa forma, o número de anos estudados e a aprendizagem continuada seriam preditores da saúde cognitiva em idosos (Banhato, 2011; Duay \& Bryan, 2008; Fillit et al., 2002; Lautenschlager, 2002; Pope et al., 2003). De acordo com Bahia e Caramelli (1998), a falta de atividades intelectuais e sociais pode causar prejuízos físicos e cognitivos, constituindo importantes indicadores de risco para declínio e para as síndromes demenciais.

Alguns mecanismos de compensação também vêm sendo utilizados com o objetivo de minimizar os prejuízos decorrentes da senescência (Yassuda, Batistoni, Fortes, \& Neri, 2006). Pesquisas demonstram a plasticidade cognitiva em idosos ao verificar que, através de treino, o indivíduo saudável é capaz de aproximar seu desempenho atual do seu desempenho máximo possível (Verhaeghen, 2000, citado por Yassuda et al., 2006). Em Oficina de Memória com duração de oito encontros de duas horas semanais, Souza e Chaves (2005) constataram melhora significativa no desempenho cognitivo entre os idosos participantes. Em consonância, em estudo de revisão bibliográfica, Fillit et al. (2002) verificaram que a atividade de treino com idosos pode melhorar várias habilidades, dentre elas o armazenamento e a recuperação da memória, a atenção e a percepção visual. Isso pode ocorrer, inclusive, entre idosos com baixa escolaridade (Lima-Silva et al., 2010) ou com CCL (Brum, Forlenza, \& Yassuda, 2009) e se manter ao longo do tempo (Aramaki, \& Yassuda, 2011).

A informática é uma das ferramentas que têm sido utilizadas para aprimorar as habilidades cognitivas de pes- 
soas na velhice. Em um trabalho de intervenção com idosos institucionalizados, McConatha, McConatha, Deaner e Dermingny (1995) verificaram que ela pode ser um instrumento efetivo para o desenvolvimento das funções cognitivas, além de terem observado que as atividades envolvidas serviram como fator de prevenção de depressão, patologia comum em pessoas residentes em instituições de longa permanência para idosos.

Em estudo transversal com duração de 14 encontros, 51 idosos participantes de uma Oficina de Inclusão Digital demonstraram ganhos significativos na velocidade de processamento de informação e na capacidade de planejamento de tarefas (Banhato et al., 2007). Ordonez, Yassuda e Cachioni (2010) observaram, também, ganhos cognitivos em idosos que participaram de um processo de inclusão digital denominado Idosos On-Line. Os autores verificaram que o grupo experimental, ao ser comparado com um controle, apresentou, no pós-teste, aumento significativo em linguagem e em memória.

Gunther, Shaefer, Holzner e Kemmler (2003) constataram que, após treino cognitivo de 14 semanas, utilizando o computador como instrumento, os idosos tiveram melhoras significativas na memória de curto e longo prazo, em tarefas que utilizavam estímulos visuais e verbais. Após cinco meses do término do programa, notou-se que esses ganhos foram mantidos. Tendo em vista essas observações, os autores sugerem que o computador pode ser usado como uma ferramenta de prevenção e tratamento de déficits cognitivos.

Considerando-se os estudos sobre informática e cognição de idosos, é possível notar que eles podem ser classificados em dois grandes grupos: (a) aqueles que utilizaram o computador como ferramenta específica para o treino cognitivo (Gunther et al., 2003); e (b) aqueles que investigaram os efeitos do uso de informática tradicional no processo cognitivo de idosos (Banhato et al., 2007; McConatha et al., 1995; Ordonez et al., 2010; Slegers, van Boxtel, \& Jolles, 2009). É importante ressaltar que a presente investigação se enquadra no segundo conjunto de pesquisas.

Seja qual for a modalidade, a utilização do computador, além de otimizar a memória de curto prazo, pode aumentar a auto-estima, encorajar a socialização e o compartilhamento de experiências e de informações (Chaffin \& Harlow, 2005; Lawhon, Ennis, \& Lawhon, 1996). Sá (2004) afirma que o domínio da tecnologia digital estimula as atividades mentais, promovendo a preservação de habilidades cognitivas e emocionais, além de poder favorecer o convívio social com indivíduos da mesma geração, muitas vezes prejudicado nessa fase da vida. Kachar (2003) assegura que a inclusão digital também pode ser uma ferramenta para o engajamento social dessa parte da população, uma vez que a dificuldade no manuseio de equipamentos digitais contribui para a desvalorização e consequente exclusão do idoso na sociedade.
O desenvolvimento e a capacidade de aprendizagem durante todas as fases da vida têm sido destacados em vários estudos, alguns deles já mencionados no presente artigo (ver, por exemplo, Scoralick-Lempke, 2009; Souza \& Chaves, 2005). Ao contrário da imagem de decadência e senilidade que o idoso possuía, verifica-se, atualmente, a possibilidade de envelhecimento ativo, participativo e saudável.

Dentre as múltiplas ferramentas que podem promover o envelhecimento saudável, o uso do computador e de outras tecnologias de informação e comunicação tem se mostrado promissor. No entanto, estudos a esse respeito ainda são escassos, principalmente na literatura brasileira. Assim, o desenvolvimento de pesquisas que avaliem a contribuição de programas que visem à manutenção e à otimização das habilidades cognitivas, emocionais e sociais, próprias de um envelhecimento saudável, deve ser uma prioridade nas áreas que estudam a senescência.

A partir da revisão de literatura, a qual sugere que o uso de informática pode promover um melhor funcionamento intelectual, o presente estudo testou a hipótese de que uma Oficina de Inclusão Digital melhoraria capacidades cognitivas de idosos, especificamente as memórias episódica e de trabalho, a metamemória e a autoeficácia em memória.

\section{Método}

\section{Participantes}

Para serem incluídos no estudo, os interessados deveriam ter idade igual ou superior a 60 anos e não serem alfabetizados em informática. Foram excluídos do estudo os idosos que apresentaram escolaridade inferior ao $6^{\circ}$ ano do ensino fundamental e/ou aqueles que não tiveram condição de acesso às dependências do INFOCENTRO do prédio da Biblioteca Central da Universidade Federal de Juiz de Fora (UFJF), local em que se realizou a pesquisa. Tais critérios se justificam, respectivamente, pelo fato de haverem atividades programadas que requeriam habilidades de leitura e escrita e, ainda, pela estrutura do campus da UFJF que possui limitações para o acesso de pessoas com incapacidade física.

Inicialmente, foram recrutados 48 idosos que foram distribuídos em três grupos, sendo dois experimentais e um controle, respeitando a sequência de listagem de inscrição. Dessa forma, os 15 primeiros integrantes da lista formaram o grupo experimental 1 (GE1), os 13 seguintes, o grupo experimental 2 (GE2) e os outros 20 constituíram o grupo controle (GC). Porém, dez deles foram excluídos do experimento em diferentes etapas do processo: seis não concluíram as Oficinas de Inclusão Digital; dois não efetuaram o pós-teste; e dois, apesar de terem feito a inscrição, não possuíam a idade mínima estipulada.

Portanto, ao final do processo de inclusão digital, o GE1 contava com 12 participantes e o GE2 com 10 , perfazen- 
do um total de 22 indivíduos no grupo experimental (GE). O GC foi constituído por 16 colaboradores. Sendo assim, o total de participantes que concluiu este estudo foi igual a 38 idosos.

A amostra foi predominantemente feminina $\left[\chi^{2}(1, \mathrm{~N}=\right.$ $38)=6,74 ; p=0,01 ; \mathrm{n}=27 ; 71,05 \%]$. Não foi verificada diferença entre o GE e o GC com relação a essa variável $\left[\chi^{2}(1, \mathrm{~N}=38)=0,98 ; p=0,32\right] .($ Tabela 1$)$

Tabela 1

Características Demográficas dos Participantes por Grupos

\begin{tabular}{lll}
\hline \multicolumn{1}{c}{ Variáveis } & \multicolumn{2}{c}{ Grupos } \\
\cline { 2 - 3 } & $\mathrm{GE}$ & $\mathrm{GC}$ \\
\hline Gênero $(\mathrm{t}, \%)$ & & \\
$\quad$ Feminino & $=17 ; 44,74 \%$ & $=10 ; 26,32 \%$ \\
$\quad$ Masculino & $=5 ; 13,16 \%$ & $=6 ; 15,79 \%$ \\
Idade $(M, \pm D P)$ & $=67,45 \pm 5,93$ & $=64,81 \pm 3,97$ \\
Escolaridade $(M, \pm D P)$ & $=9,64 \pm 3,72$ & $=9,81 \pm 3,90$ \\
\hline
\end{tabular}

A idade média dos colaboradores em anos foi de $66,34 \pm 5,30$. Ao realizar a análise inter grupos, verificouse que o GE e o GC eram equivalentes $[\mathrm{t}(36)=1,55 ; p=$ $0,13]$. Em relação à escolaridade, a média de anos estudados foi de $9,71 \pm 3,74$. Também não foi constatada diferença significativa entre o $\mathrm{GC}$ e o GE $[\mathrm{t}(36)=0,14 ; p=$ $0,89]$ nessa variável.

\section{Materiais}

No pré-teste, utilizou-se um questionário para coletar dados demográficos dos participantes. Para avaliação da cognição global, da memória, da metamemória e da autoeficácia em memória, foram usados tanto no pré quanto no pós-teste, os seguintes instrumentos:

1. MEEM - Mini Exame do Estado Mental (Folstein, Folstein, \& McHugh, 1975): utilizado para a avaliação da cognição global, permite rastrear possíveis casos de declínio cognitivo. É formado por 30 itens que avaliam orientação tempo-espacial, memória imediata, linguagem, memória episódica, atenção e cálculo, habilidade visoconstrutiva. O resultado deste instrumento consiste na soma dos acertos. Neste estudo, foi utilizado o ponto de corte 23/24 para amostras sem escolaridade ou com alguma escolaridade, respectivamente, sugerido por Almeida (1998).

2. Lista de palavras e História: desenvolvidas, respectivamente, por West e Thorn (2001) e por Dixon, Hultsch e Hertzog (1989) e adaptadas para o Brasil por Yassuda et al. (2005), utilizadas para avaliar a memória episódica. A Lista de Palavras é composta por 35 termos, os quais o indivíduo tem cinco minutos para tentar memorizar e, posteriormente, três minutos para escrever as palavras recordadas. Cada palavra lembrada consiste em um ponto, sendo obtido o máximo de 35 pontos. A História, por sua vez, é composta por 63 ideias e o sujeito é instruído a estudá-la por cinco minutos e, em seguida, redigir as sentenças lembradas, também no mesmo período de tempo. São pontuadas as sentenças que se assemelham às ideias contidas no texto. A pontuação pode variar de zero a 63 pontos. Adotou-se o procedimento de análise que envolvia dois juízes que avaliaram as histórias e, em caso de discordância, um terceiro juiz atribuiu a nota final.

3. Subteste Dígitos da escala WAIS-III (Wechsler, 1997, adaptado por Nascimento, 2000): usado para avaliar a memória de trabalho. Este instrumento investiga as habilidades de recordação e repetição imediata, consistindo na repetição oral de sequências numéricas em ordem direta (16 itens) e inversa (14 itens), perfazendo 30 pontos. A pontuação é obtida através da soma dos itens acertados. Neste estudo, a variável de interesse foi o número de acertos da sequência numérica na ordem inversa, que avalia diretamente a memória operacional.

4. MIA - Metamemory Adulthood Questionnaire (Dixon \& Hultsch, 1983 adaptado por Yassuda et al., 2005): avalia a metamemória em idosos. É composto por 108 itens, cada um com cinco possibilidades de resposta. $\mathrm{O}$ instrumento analisa sete dimensões da metamemória: estratégia, tarefa, capacidade, mudança, ansiedade, metas e controle. $\mathrm{O}$ resultado do instrumento é obtido através da soma dos pontos das sete dimensões.

5. MSEQ - Memory Self Efficacy Questionnaire (Berry et al., 1989 adaptado por Yassuda et al., 2005): utilizado para avaliar a autoeficácia em memória. Os participantes são solicitados a avaliar quão bem poderiam realizar uma tarefa de memória dentro de cinco graus de possibilidade, em seis subescalas: lista, história, objetos, nomes de pessoas, coisa a fazer e palavras. O resultado deste instrumento é obtido em dois âmbitos: (a) o nível, que representa a quantidade de respostas positivas em relação à tarefa proposta; e (b) a força, que representa o grau de confiança que o indivíduo tem em conseguir realizar determinada atividade. Para efetuar a correção do instrumento, soma-se a quantidade de itens em que o indivíduo respondeu "sim" em cada subescala para ser obtido o Nível de autoeficácia (NAE) em memória. Para avaliar a Força (FAE), faz-se uma média das porcentagens que a pessoa marcou em cada item das subescalas.

\section{Procedimento}

Após aprovação do Comitê de Ética em Pesquisa da UFJF (CAAE- 0064.0.180.000-07), os idosos inscritos para compor o GE foram contatados por telefone para o 
agendamento da assinatura do Termo de Consentimento Livre e Esclarecido e, tendo concordado com o estudo, foi realizado o pré-teste.

As atividades do pré-teste foram realizadas em um único dia. A Lista de Palavras (Yassuda et al., 2005), a História (Yassuda et al., 2005) e o MSEQ (Yassuda et al., 2005) foram aplicados coletivamente, em grupos de aproximadamente 15 pessoas. Um pesquisador lia as instruções em voz alta e, em seguida, os participantes eram solicitados a realizar a atividade. Durante toda a avaliação, quatro estudantes, adequadamente treinados, percorreram a sala, auxiliando os participantes, quando eram solicitados. O MEEM (M. F. Folstein et al., 1975) e o Subteste Dígitos da escala WAIS-III (Nascimento, 2000) foram aplicados individualmente, após as avaliações coletivas. O tempo de duração aproximado para a realização de todas as atividades foi de uma hora e 20 minutos. O MIA (Yassuda et al., 2005) foi entregue aos idosos no dia do pré-teste, os quais foram instruídos a responderem-no em casa e retornarem como ele no primeiro dia da Oficina. Esse procedimento foi adotado devido à extensão do instrumento. Foi exposto que, caso os participantes tivessem alguma dúvida em responder o MIA, eles deveriam deixar a questão em branco para a ajuda dos tutores no início do primeiro dia de aula.

Os mesmos procedimentos foram realizados com os sujeitos do GC. No entanto, a instrução foi a de entregar o instrumento MIA em local pré-definido na semana seguinte à realização do pré-teste. Por questões éticas, os participantes desse grupo foram informados de que iriam começar a Oficina de Inclusão Digital cinco meses após a data da primeira avaliação.

As Oficinas de Inclusão Digital foram ministradas por uma acadêmica do curso de Psicologia, com o auxílio de uma Psicóloga e de quatro estudantes do referido curso, que atuaram como tutores. Os GEs participaram de 19 encontros distribuídos ao longo de cinco meses. Eles foram realizados uma vez por semana, cada um com duração de duas horas. Os seguintes conteúdos foram abordados: ambiente Windows, Paint, Microsoft Word, Microsoft PowerPoint e Internet Explorer.

Tabela 2

Médias e Desvios Padrão nas Medidas Pré e Pós-Testes por Grupo

\begin{tabular}{|c|c|c|c|c|}
\hline \multirow[t]{3}{*}{ Instrumentos } & \multicolumn{4}{|c|}{ Grupos/Momento } \\
\hline & \multicolumn{2}{|c|}{ Grupo Experimental } & \multicolumn{2}{|c|}{ Grupo Controle } \\
\hline & Pré-teste & Pós-teste & Pré-teste & Pós-teste \\
\hline MEEM & $28,86 \pm 0,94$ & $28,82 \pm 1,22$ & $28,31 \pm 1,20$ & $28,19 \pm 1,97$ \\
\hline Dígitos (ordem direta) & $9,18 \pm 2,65$ & $9,64 \pm 2,77$ & $8,75 \pm 2,79$ & $9,50 \pm 2,73$ \\
\hline Dígitos (ordem inversa) & $5,14 \pm 1,91$ & $5,68 \pm 2,48$ & $4,94 \pm 2,02$ & $5,63 \pm 1,45$ \\
\hline Lista de Palavras* & $11,77 \pm 2,60$ & $14,05 \pm 3,37$ & $11,80 \pm 3,69$ & $14,33 \pm 4,17$ \\
\hline História* & $24,50 \pm 8,32$ & $27,00 \pm 7,39$ & $21,93 \pm 10,34$ & $26,00 \pm 10,21$ \\
\hline MIA**: Estratégia & $59,48 \pm 10,91$ & $62,48 \pm 11,68$ & $57,29 \pm 6,17$ & $56,87 \pm 10,35$ \\
\hline MIA**: Tarefa & $64,95 \pm 9,57$ & $62,95 \pm 7,44$ & $62,58 \pm 6,19$ & $60,13 \pm 7,35$ \\
\hline MIA**: Capacidade & $54,00 \pm 11,52$ & $52,19 \pm 12,11$ & $54,75 \pm 9,44$ & $53,07 \pm 11,48$ \\
\hline MIA**: Mudança & $48,52 \pm 11,54$ & $47,19 \pm 12,57$ & $46,36 \pm 9,58$ & $46,47 \pm 9,85$ \\
\hline MIA**: Ansiedade & $47,14 \pm 12,05$ & $46,95 \pm 10,12$ & $46,38 \pm 11,10$ & $45,40 \pm 10,05$ \\
\hline MIA**: Metas $^{*}$ & $65,52 \pm 6,11$ & $64,86 \pm 5,64$ & $66,80 \pm 4,42$ & $64,87 \pm 4,27$ \\
\hline MIA $^{* *}:$ Controle & $34,10 \pm 4,07$ & $31,62 \pm 4,07$ & $33,02 \pm 4,41$ & $33,67 \pm 4,35$ \\
\hline NAE Lista de supermercado & $3,23 \pm 0,87$ & $3,59 \pm 1,14$ & $3,38 \pm 1,09$ & $3,44 \pm 1,36$ \\
\hline NAE História & $3,23 \pm 1,11$ & $3,09 \pm 1,38$ & $2,81 \pm 1,33$ & $3,13 \pm 1,59$ \\
\hline NAE Localização de objetos & $3,45 \pm 1,10$ & $3,23 \pm 1,51$ & $3,25 \pm 1,00$ & $3,25 \pm 1,57$ \\
\hline NAE Nome de pessoas & $4,00 \pm 1,23$ & $3,32 \pm 1,39$ & $3,13 \pm 1,50$ & $3,56 \pm 1,67$ \\
\hline NAE Lista de coisas a fazer & $3,14 \pm 0,99$ & $3,09 \pm 1,27$ & $2,63 \pm 0,72$ & $3,06 \pm 1,34$ \\
\hline NAE Lista de palavras & $3,95 \pm 0,95$ & $3,68 \pm 1,13$ & $3,13 \pm 0,96$ & $3,94 \pm 1,06$ \\
\hline NAE Total & $3,50 \pm 0,74$ & $3,33 \pm 1,11$ & $3,05 \pm 0,75$ & $3,40 \pm 1,20$ \\
\hline FAE Lista de supermercado & $80,82 \pm 19,19$ & $76,00 \pm 18,23$ & $70,68 \pm 25,90$ & $71,94 \pm 14,37$ \\
\hline FAE História & $74,99 \pm 23,53$ & $70,00 \pm 24,72$ & $59,35 \pm 28,00$ & $56,25 \pm 20,20$ \\
\hline FAE Localização de objetos & $74,10 \pm 22,38$ & $68,82 \pm 28,76$ & $58,06 \pm 27,62$ & $62,31 \pm 21,25$ \\
\hline FAE Nome de pessoas & $79,95 \pm 15,25$ & $75,14 \pm 19,72$ & $68,95 \pm 24,69$ & $64,31 \pm 19,80$ \\
\hline FAE Lista de coisas a fazer & $77,12 \pm 23,21$ & $71,64 \pm 19,57$ & $65,35 \pm 13,40$ & $68,00 \pm 16,57$ \\
\hline FAE Lista de palavras & $84,94 \pm 14,01$ & $74,36 \pm 19,81$ & $68,31 \pm 18,81$ & $64,63 \pm 17,63$ \\
\hline FAE Total & $78,65 \pm 17,09$ & $72,66 \pm 19,24$ & $65,12 \pm 18,12$ & $64,57 \pm 14,53$ \\
\hline
\end{tabular}

Notas. *Um participante do GE não respondeu $(n=21)$ **Um participante do GE e um do GC não responderam $(\mathrm{GE}: \mathrm{n}=21 ; \mathrm{GC}: \mathrm{n}=15)$. 
Scoralick-Lempke, N. N., Barbosa, A. J. G. \& Mota, M. M. P. E. (2012). Efeitos de um Processo de Alfabetização em Informática na Cognição de Idosos.

As atividades foram divididas em dois momentos durante as oficinas, e envolviam: (a) explicação teórica e contextualização da utilidade dos programas; e (b) aplicação prática, com a realização de atividade para a fixação do conteúdo. Ao início de cada oficina, o conteúdo abordado no encontro anterior era relembrado por meio de atividades de revisão. Realizou-se, ainda, uma visita a uma Lan House para que os participantes conhecessem o funcionamento destas lojas. Essa atividade almejou assegurar que, após as Oficinas, os participantes que não possuíssem acesso ao computador tivessem uma opção de acesso a essa tecnologia.

No último dia de Oficina, fez-se o agendamento do pós-teste com os GEs para verificar os resultados do processo de alfabetização digital. O instrumento MIA foi entregue no último encontro, sendo pedido que os participantes respondessem e entregassem no pós-teste. As demais avaliações foram realizadas da mesma forma que no pré-teste.

No mesmo período, os participantes do GC foram contatados por telefone para o agendamento e realização do pós-teste, a fim de compará-los com os grupos que tiveram a intervenção (GE). As avaliações foram realizadas da mesma forma que no pré-teste.

\section{Resultados}

A Tabela 2 apresenta a estatística descritiva referente aos 26 processos cognitivos que foram alvo da presente investigação para GE e GC. A partir das médias, foram efetuadas comparações intra e inter grupos, mais especificamente, realizaram-se análises de variância fatorial com tempo como fator de medidas repetidas e grupo como fator inter participantes. Considerou-se um nível de significância de 0,05 por omissão.

A partir dessas análises, foram obtidos resultados significantes para o subteste do WAIS-III Dígitos - Ordem Inversa $[\mathrm{F}(1,36)=4,49 ; \mathrm{p}<0,05]$, Lista de Palavras $[F(1,35)=18,77 ; \mathrm{p}<0,05]$ e História $[\mathrm{F}(1,35)=8,43 ; \mathrm{p}$ $<0,05]$ para variável tempo (Tabela 3 ). Nesses três casos, os escores dos dois grupos melhoraram do pré-teste para o pós-teste. Houve um efeito significativo para variável tempo na análise feita com os escores no teste FAE Lista de Palavras $[\mathrm{F}(1,36)=4,71 ; \mathrm{p}<0,05]$, mas, nesse caso, os grupos pioraram.

Tabela 3

Análises de Variância Fatoriais com Tempo como Fator de Medidas Repetidas e Grupo como Fator Inter Participantes

\begin{tabular}{llll}
\hline Medidas & Grupo & Tempo & Grupo/Tempo \\
\hline MEEM & $\mathrm{F}(1,36)=2,44$ & $\mathrm{~F}(1,36)=0,14$ & $\mathrm{~F}(1,36)=0,03$ \\
Dígitos (ordem direta) & $\mathrm{F}(1,36)=0,13$ & $\mathrm{~F}(1,36)=1,75$ & $\mathrm{~F}(1,36)=0,11$ \\
Dígitos (ordem inversa) & $\mathrm{F}(1,36)=0,05$ & $\mathrm{~F}(1,36)=4,49^{*}$ & $\mathrm{~F}(1,36)=0,06$ \\
Lista de Palavras & $\mathrm{F}(1,35)=0,02$ & $\mathrm{~F}(1,35)=18,77^{*}$ & $\mathrm{~F}(1,35)=0,06$ \\
História & $\mathrm{F}(1,35)=0,42$ & $\mathrm{~F}(1,35)=8,43^{*}$ & $\mathrm{~F}(1,35)=0,48$ \\
MIA: Estratégia & $\mathrm{F}(1,34)=1,47$ & $\mathrm{~F}(1,34)=1,00$ & $\mathrm{~F}(1,34)=1,76$ \\
MIA: Tarefa & $\mathrm{F}(1,34)=1,18$ & $\mathrm{~F}(1,34)=3,55$ & $\mathrm{~F}(1,34)=0,04$ \\
MIA: Capacidade & $\mathrm{F}(1,34)=0,05$ & $\mathrm{~F}(1,34)=2,47$ & $\mathrm{~F}(1,34)=0,00$ \\
MIA: Mudança & $\mathrm{F}(1,34)=0,16$ & $\mathrm{~F}(1,34)=0,29$ & $\mathrm{~F}(1,34)=0,40$ \\
MIA: Ansiedade & $\mathrm{F}(1,34)=0,11$ & $\mathrm{~F}(1,34)=0,39$ & $\mathrm{~F}(1,34)=0,18$ \\
MIA: Metas & $\mathrm{F}(1,34)=0,17$ & $\mathrm{~F}(1,34)=2,14$ & $\mathrm{~F}(1,34)=0,51$ \\
MIA: Controle & $\mathrm{F}(1,34)=0,16$ & $\mathrm{~F}(1,34)=1,66$ & $\mathrm{~F}(1,34)=4,80^{*}$ \\
NAE Lista de supermercado & $\mathrm{F}(1,36)=0,00$ & $\mathrm{~F}(1,36)=1,32$ & $\mathrm{~F}(1,36)=0,66$ \\
NAE História & $\mathrm{F}(1,36)=0,22$ & $\mathrm{~F}(1,36)=0,26$ & $\mathrm{~F}(1,36)=1,68$ \\
NAE Localização de objetos & $\mathrm{F}(1,36)=0,06$ & $\mathrm{~F}(1,36)=0,24$ & $\mathrm{~F}(1,36)=0,24$ \\
NAE Nome de pessoas & $\mathrm{F}(1,36)=0,65$ & $\mathrm{~F}(1,36)=0,22$ & $\mathrm{~F}(1,36)=4,51^{*}$ \\
NAE Lista de coisas a fazer & $\mathrm{F}(1,36)=0,78$ & $\mathrm{~F}(1,36)=0,97$ & $\mathrm{~F}(1,36)=1,47$ \\
NAE Lista de palavras & $\mathrm{F}(1,36)=1,04$ & $\mathrm{~F}(1,36)=2,08$ & $\mathrm{~F}(1,36)=8,39^{*}$ \\
NAE Total & $\mathrm{F}(1,36)=0,46$ & $\mathrm{~F}(1,36)=0,38$ & $\mathrm{~F}(1,36)=3,12$ \\
FAE Lista de supermercado & $\mathrm{F}(1,36)=1,98$ & $\mathrm{~F}(1,36)=0,19$ & $\mathrm{~F}(1,36)=0,57$ \\
FAE História & $\mathrm{F}(1,36)=4,65^{*}$ & $\mathrm{~F}(1,36)=0,96$ & $\mathrm{~F}(1,36)=0,05$ \\
FAE Localização de objetos & $\mathrm{F}(1,36)=2,99$ & $\mathrm{~F}(1,36)=0,01$ & $\mathrm{~F}(1,36)=0,86$ \\
FAE Nome de pessoas & $\mathrm{F}(1,36)=4,17^{*}$ & $\mathrm{~F}(1,36)=1,65$ & $\mathrm{~F}(1,36)=0,00$ \\
FAE Lista de coisas a fazer & $\mathrm{F}(1,36)=2,09$ & $\mathrm{~F}(1,36)=0,19$ & $\mathrm{~F}(1,36)=1,53$ \\
FAE Lista de palavras & $\mathrm{F}(1,36)=7,66^{*}$ & $\mathrm{~F}(1,36)=4,71^{*}$ & $\mathrm{~F}(1,36)=1,10$ \\
FAE Total & $\mathrm{F}(1,36)=4,86^{*}$ & $\mathrm{~F}(1,36)=1,19$ & $\mathrm{~F}(1,36)=0,83$ \\
\hline & & &
\end{tabular}

$* \mathrm{p}<0,05$. 
As interações entre grupo e tempo foram significativas para os testes MIA Controle $[\mathrm{F}(1,34)=4,80 ; \mathrm{p}<0,05]$, NAE Nome de Pessoas $[\mathrm{F}(1,36)=4,51 ; \mathrm{p}<0,05]$ e NAE Lista de Palavras $[\mathrm{F}(1,36)=8,39 ; \mathrm{p}<0,05]$. Análises post hoc foram realizadas por meio de testes t com ajuste do nível de significância, usando o critério de Bonferroni.

Para MIA Controle, observou-se, por meio do teste $\mathrm{t}$ para amostra pareada, que o GC se manteve estável do pré para o pós-teste $[\mathrm{t}(14)=-0,62 ; \mathrm{p}=0,55]$ e que $\mathrm{GE}$ piorou $[\mathrm{t}(20)=2,63 ; \mathrm{p}=0,016]$. Nesse caso, o valor ajustado de p, com o critério de Bonferroni, foi 0,025.

A análise post hoc revelou que, para o NAE Nome de Pessoas, não foi identificada diferença significante quando foi utilizado o critério de Bonferroni $(\mathrm{p}<0,017)$. Obtiveram-se valores do Teste $\mathrm{t}$ das amostras pareadas iguais a 2,14 $(\mathrm{gl}=21 ; \mathrm{p}=0,044)$ e $-1,00(\mathrm{gl}=15 ; \mathrm{p}=$ 0,33) para, respectivamente, GE e GC. Foi efetuado, também, o Teste $\mathrm{t}[\mathrm{t}(36)=1,97 ; \mathrm{p}=0,057]$ para amostras independentes, e não foi observada diferença estatisticamente significativa entre os subgrupos no préteste.

Ao efetuar o post hoc do NAE Lista de Palavras $(\mathrm{p}<$ 0,017 ajustado para Bonferroni), foram observadas diferenças significativas tanto ao parear o pré e o pós-teste do $\mathrm{GC}[\mathrm{t}(15)=-2,93 ; \mathrm{p}=0,01]$, quanto ao comparar GE e $\mathrm{GC}$ no pré-teste $[\mathrm{t}(36)=2,65 ; \mathrm{p}=0,01]$. O pareamento pré e pós-teste para GE não apresentou diferença significante $[\mathrm{t}(21)=1,10 ; \mathrm{p}=0,28]$. Portanto, GE possuía um desempenho superior ao de GC no pré-teste, mas, ao final do experimento, ambos apresentaram autoeficácia para recordar palavras equivalentes, devido ao crescimento desse último subgrupo.

\section{Discussão}

Criar estratégias de prevenção e promoção da saúde na velhice é, atualmente, uma prioridade mundial. De acordo com Ramos (2003), o estado cognitivo e o grau de dependência no dia a dia são os únicos fatores que podem sofrer intervenções a fim de diminuir a morbidade e a mortalidade em idosos. Dessa forma, a prevenção dos déficits intelectuais assume um caráter importante na longevidade, na autonomia e na qualidade de vida dessa população.

Dentre as múltiplas intervenções que podem preservar e/ou promover as cognições de idosos, o emprego de informática tem se mostrado promissor. A literatura científica (Slegers et al., 2009) destaca que o computador representa uma alternativa para prevenir o declínio cognitivo na velhice, sendo que essa tecnologia pode ser empregada tanto no treino cognitivo, quanto em processos educacionais mais amplos, os quais almejam que esse artefato passe a fazer parte do cotidiano dos idosos, inclusive para o exercício da cidadania. Há que se ressaltar que essa última modalidade de emprego da informática esbarra no imenso analfabetismo digital que assola a população de idosos no Brasil.
Tendo em vista essa necessidade e considerando a importância da temática, elaborou-se um processo de alfabetização em informática para essa coorte etária, aqui denominado Oficina de Inclusão Digital, em que foram avaliados os efeitos das atividades em certas capacidades cognitivas desses indivíduos. Os resultados evidenciam que as alterações nos processos cognitivos dos participantes, observadas na presente investigação, não podem ser atribuídas a esse processo educacional, uma vez que foram observadas interações entre tempo e grupo em somente três das 26 variáveis analisadas. Além disso, há que se considerar que, em uma delas - NAE Lista de Palavras -, GC apresentou nível de autoeficácia mais elevado no pós-teste, GE piorou em outra - MIA Controle - e não foi possível por meio do post hoc precisar o que ocorreu com uma terceira variável - NAE Nome de Pessoas. Portanto, trata-se de associações espúrias entre variáveis.

Não se pode afirmar, porém, que o uso de computadores não influencia a cognição humana, seja na velhice ou em outra etapa do curso de vida. Todavia, parece que a influência pode ocorrer a partir do uso intenso, frequente e duradouro dessa tecnologia, circunstância que não aconteceu com os participantes desta pesquisa. De fato, os idosos foram submetidos a um processo inicial de aprendizagem de informática, com uma carga horária bastante modesta, a qual foi distribuída ao longo de um intervalo de tempo relativamente curto.

Essas e outras limitações do estudo exigem cuidados na interpretação dos resultados. Há que se mencionar o processo amostral como uma circunscrição da investigação, pois a amostra foi obtida de forma não probabilística e foi distribuída dessa mesma forma nos grupos (GE e GC). Acrescenta-se nisso o fato de que parte dos instrumentos usados ainda demanda mais evidências de validade para idosos brasileiros. Ademais, é preciso considerar que somente a participação em processos de alfabetização digital foi controlada para o GC, não sendo controlada, portanto, a realização de outras atividades educacionais que poderiam ter gerado a melhoria em algumas variáveis.

Assim, os resultados desta pesquisa não devem ser interpretados como indicadores de que atividades educacionais não favorecem o desenvolvimento cognitivo na velhice e de que o uso de computadores não gera ganhos intelectuais nessa fase do curso de vida. Ao contrário, há uma literatura bastante farta que evidencia o fato de as atividades intelectuais serem fatores protetores de declínio cognitivo no envelhecimento (ver, por exemplo, Bahia \& Caramelli, 1998; Banhato, 2011; Gage, 2003).

Quando se tratam dos benefícios cognitivos do uso de informática por idosos, entretanto, a produção científica não é tão ampla e, muito menos, conclusiva. Coexistem estudos, como o de Banhato et al. (2007), J. T. McConatha et al. (1995) e Ordonez et al. (2010), que obtiveram resultados bastante favoráveis e outros, como, 
por exemplo, o realizado por Slegers et al. (2009), que chegaram a resultados bem menos abonadores. Tais divergêncvias podem ter relação com os sujeitos estudados, intervenções realizadas e instrumentos utilizados, por exemplo.

Voltando a outro resultado relevante da presente pesquisa, o MEEM revelou serem os participantes cognitivamente saudáveis, pois obtiveram escores acima do ponto de corte. Os resultados obtidos em outras medidas, ainda que não possuam normas ou pontos de corte, denotam, também, que a amostra tinha as capacidades cognitivas avaliadas preservadas. Assim, não se tratava de um grupo de idosos 'normais' e muito menos com declínio cognitivo. No pré-teste, eles já possuíam uma linha de base bastante alta nas várias medidas, o que pode ter limitado a capacidade de o experimento - Oficinas de Inclusão Digital - promover ganhos para o GE nos processos cognitivos observados. Desse modo, sugere-se que outras pesquisas análogas a esta, mas envolvendo indivíduos com algum declínio ou residentes em instituições de longa permanência para idosos, como na investigação de J. T. McConatha et al. (1995), sejam realizadas para que se conheçam os efeitos da alfabetização em informática nesse tipo de população.

$\mathrm{O}$ experimento realizado aponta para o fato de que o processo de alfabetização em informática pode ter servido para o GE mais como um mecanismo de prevenção de déficit cognitivo em idosos saudáveis. No entanto, estudos longitudinais devem ser realizados para corroborar essa hipótese. Apenas esse delineamento permitirá verificar os benefícios em médio e em longo prazo da aprendizagem de informática em processos cognitivos.

Para futuras pesquisas, destaca-se, também, a necessidade de que o uso de informática com idosos seja baseado em sólidos princípios de Gerontologia Educacional. Só assim, os computadores serão, de fato, mídias cognitivas. Uma mídia cognitiva é aquela que propicia um ambiente para crescimento intelectual (Hokanson \& Hooper, 2000).

\section{Referências}

Abreu, I. D., Forlenza, O. V., \& Barros, L. H. (2005). Demência de Alzheimer: Correlação entre memória e autonomia. Revista de Psiquiatria Clínica, 32(3), 131-136.

Almeida, O. P. (1998). Queixa de problemas com a memória e o diagnóstico de demência. Arquivos de Neuropsiquiatria, 56(3-A), 412-418.

American Psychiatric Association. (1995). Manual diagnóstico e estatístico de transtornos mentais - DSM IV (4. ed.). Porto Alegre, RS: Artes Médicas.

Antunes, H. K. M., Santos, R. F., Cassilhas, R., Santos, R. V. T., Bueno, O. F. A., \& Mello, M. T. (2006, março/abril). Exercício físico e função cognitiva: Uma revisão. Revista Brasileira de Medicina do Esporte, 12(2), 108-114.

Aramaki, F. O., \& Yassuda, M. S. (2011). Cognitive training based on metamemory and mental images: Follow-up evaluation and booster training effects. Dementia \& Neuropsychologia, 5(1), 48-53.
Argimon, I. I. A., \& Stein, L. M. (2005). Habilidades cognitivas em indivíduos muito idosos: Um estudo longitudinal. Caderno de Saúde Pública, 21(1), 64-72.

Baddeley, A. D. (2000). The episodic buffer: A new component of working memory? Trends in Cognitive Science, 4(11), 417-423.

Bahia, V. S., \& Caramelli, P. (1998). Prevenção do declínio cognitivo relacionada à idade. In W. Jacob Filho \& E. T. Carvalho (Eds.), Promoção da saúde do idoso (pp. 45-53). São Paulo, SP: Lemos.

Baltes, P. B. (1987). Theoretical propositions of life-span developmental psychology: On the dynamics between growth and decline. Developmental Psychology, 32(5), 611-626.

Bandura, A. (1977). Self-efficacy: Toward a unifiyng theory of behavioral change. Psycological Review, 85, 191-215.

Banhato, E. F. C. (2011). Validade de critério de uma forma abreviada da escala WAIS III em uma amostra de idosos brasileiros. (Tese de Doutorado não-publicada). Programa de Pós-Graduação em Saúde Brasileira, Universidade Federal de Juiz de Fora, MG.

Banhato, E. F. C., Atalaia-Silva, K. C., Magalhaes, N. C., Mota, M. M. P. E., Guedes, D. V., \& Scoralick, N. N. (2007). Inclusão digital: Ferramenta de promoção de envelhecimento saudável? Trabalho apresentado no IV Congresso Interamericano de Psicologia da Saúde: Neurociências e Psicopatologia Interfaces, São Paulo, SP.

Bottino, C. M. C., \& Moreno, M. D. P. (2006). Comprometimento cognitivo leve: Critérios diagnósticos e validade clínica. In. M. C. Bottino, J. Laks, \& S. L. Blay (Eds.), Demência e transtornos cognitivos em idoso (pp. 31-37). Rio de Janeiro, RJ: Guanabara Koogan.

Brum, P. S., Forlenza, O. V., \& Yassuda, M. S. (2009). Cognitive training in older adults with mild cognitive impairment: Impact on cognitive and functional performance. Dementia \& Neuropsychologia, 3(2), 124-131.

Carvalho, F. C. R., Neri, A. L., \& Yassuda, M. S. (2010). Treino de memória episódica com ênfase em categorização para idosos sem demência e depressão. Psicologia: Reflexão e Crítica, 23(2), 317-323.

Chaffin, A. M., \& Harlow, S. D. (2005). Cognitive learning applied to older adult learners and technology. Educational Gerontology, 31, 301-329.

Damasceno, B. P. (1999). Envelhecimento cerebral: O problema dos limites entre o normal e o patológico. Arquivos de Neuropsiquiatria, 57(1), 78-83.

Duay, D. L., \& Bryan, V. C. (2008). Learning in later life: What seniors want in a learning experience. Educational Gerontology, 34, 1070-1086.

Engelhardt, E., Laks, J., Rozenthal M., \& Marinho, V. M. (1998). Idosos institucionalizados: Rastreamento cognitivo. Revista de Psiquiatria Clínica, 25(2), 74-79.

Fillit, H. M., Butler, R. N., O'Connell, A. W., Albert, M. S., Birren, J. E., Cotman, C. W., et al. (2002). Achieving and maintaining cognitive vitality with aging. Mayo Clinic Proceedings, 77, 681-696.

Folstein, M. F., Folstein, S. E., \& McHugh, P. R. (1975). Minimental state: A practical method for grading the cognitive state of patients for the clinicians. Journal of Psychiatric Research, 12, 189-198.

Gage, F. H. (2003) A auto recuperação cerebral. Scientific American, 17, 40-47. 
Gunther, V. K., Shaefer, P., Holzner, B. J., \& Kemmler, G. W. (2003). Long term improvimentes in cognitive performance trough computer-assisted cognitive training: A pilot study in a residential home for de older people. Aging and Mental Health, 7(3), 200-206.

Hamdan, A. C. (2008). Avaliação neuropsicológica na doença de Alzheimer e no comprometimento cognitivo leve. Psicologia Argumento, 26(54), 183-192.

Hokanson, B., \& Hooper, S. (2000). Computers as cognitive media: Examining the potential of computers in education. Computers in Human Behavior, 16, 537-552.

Kachar, V. (2003). Terceira idade \& informática: Aprender revelando potencialidades. São Paulo, SP: Cortez.

Lautenschlager, N. T. (2002). Is it possible to prevent dementia? Revista Brasileira de Psiquiatria, 24, 22-27.

Lawhon, T., Ennis, D., \& Lawhon, D. C. (1996). Senior adults and computers in the 1990s [Abstract]. Educational Gerontology, 22(2), 193-201.

Lima-Silva, T. B., Ordonez, T. N., Santos, G. D., Fabrício, A. T., Aramaki, F. O., Almeida, E. B., et al. (2010). Effects of cognitive training based on metamemory and mental images. Dementia \& Neuropsychologia, 4(2), 114-119.

Lombroso, P. (2004). Aprendizado e memória. Revista Brasileira de Psiquiatria, 26(3), 207-210.

Mattos, P., Lino, V., Rizo, L., Alfano, A., Araújo, C., \& Raggio, R. (2003). Memory complaints and teste performance in healthy elderly persons. Arquivos de. Neuropsiquiatria, 61(4), 920-924.

McConatha, J. T., McConatha, D., Deaner, S. L., \& Dermingny, R. (1995). A computer-based intervention for the education and therapy of institutionalized older adults [Abstract]. Educational Gerontology, 21(2), 129-138.

Nascimento, E. (2000). Adaptação e validação do teste WAISIII para um contexto brasileiro. (Tese de Doutorado nãopublicada). Curso de Pós-Graduação em Psicologia, Universidade de Brasília, DF.

Ordonez, T. N., Yassuda, M. S., \& Cachioni, M. (2010, December). Elderly online: Effects of a digital inclusion program in cognitive performance. Archives of Gerontology and Geriatrics. Retrieved June 18, 2011, from http://www. elsevier.com/locate/archger

Parente, M. A. M. P., \& Wagner, G. P. (2006). Teorias abrangentes sobre o envelhecimento cognitivo. In M. A. M. P. Parente (Ed.), Cognição e envelhecimento (pp. 31-46). Porto Alegre, RS: Artmed.

Pope, S. K., Shue, V. M., \& Beck, C. (2003). Will a healthy lifestyle help prevent Alzheimer's disease? Annual Review of Public Health, 24, 111-132.

Ramos, L. R. (2003). Fatores determinantes do envelhecimento saudável em idosos residentes em centro urbano: Projeto Epidoso, São Paulo. Caderno de Saúde Pública, 19(3), 793798.

Sá, J. L. M. (2004). Educação e envelhecimento. In L. Py, J. L. Pacheco, J. L. M. Sá, \& S. N. Goldman, Tempo de envelhecer: Percursos e dimensões psicossociais. Rio de Janeiro, RJ: Nau.

Salthouse, T. A. (1984). Effects of age and skill in typing. Journal of Experimental Psychology General, 13, 345-371.

Scoralick-Lempke, N. N (2009). Alfabetização digital: Aprendizagem e utilização do computador na velhice. (Dissertação de Mestrado não-publicada). Programa de Pós-Graduação em Psicologia, Universidade Federal de Juiz de Fora, MG.
Shacter, D. L. (2003). Os sete pecados da memória: Como a mente esquece e lembra. Rio de Janeiro, RJ: Rocco.

Slegers, K., van Boxtel, M., \& Jolles, J. (2009). The effects of computer training and Internet usage on cognitive abilities of older adults: A randomised controlled study. Aging Clinical and Experimental Research, 21(1), 43-54.

Souza, J. N., \& Chaves, E. C. (2005). O efeito do exercício de estimulação da memória em idosos saudáveis. Revista da Escola de Enfermagem da USP, 39(1), 13-19.

Taussik, I., \& Wagner, G. P. (2006). Memória explícita e envelhecimento. In M. A. M. P. Parente (Ed.), Cognição e envelhecimento (pp. 67-84). Porto Alegre, RS: Artmed.

Yassuda, M. S., Batistoni, S. S. T., Fortes, A. G., \& Neri, A. L. (2006). Treino de memória no idoso: Benefícios e mecanismos. Psicologia: Reflexão e Crítica, 19(3), 470-481.

Yassuda, M. S., Lasca, V. B., \& Neri, A. L. (2005). Meta-memória e auto-eficácia: Um estudo de validação de instrumentos de pesquisa sobre memória e envelhecimento. Psicologia: Reflexão e Crítica, 18(1), 78-90.
Recebido: 30/03/2011

$1^{a}$ revisão: 07/07/2011 Aceite final: 02/09/2011 